\title{
Optimization of the Preparation Process for an Oral Phytantriol-Based Amphotericin B Cubosomes
}

\author{
Zhiwen Yang, ${ }^{1,2}$ Xinsheng Peng, ${ }^{1,3}$ Yinhe Tan, ${ }^{1}$ Meiwan Chen, ${ }^{1}$ Xingqi Zhu, ${ }^{4}$ Min Feng, ${ }^{1}$ \\ Yuehong $\mathrm{Xu},{ }^{1}$ and Chuanbin $\mathrm{Wu}^{1,5}$ \\ ${ }^{1}$ School of Pharmaceutical Sciences, Sun Yat-Sen University, Guangzhou 51006, China \\ ${ }^{2}$ Department of Pharmaceutical Sciences, Gannan Medical College, Ganzhou, China \\ ${ }^{3}$ GuangDong Medical College, Dongguan, China \\ ${ }^{4}$ Anton-Paar, Shanghai, China \\ ${ }^{5}$ GuangDong Research Center for Drug Delivery Systems, Guangzhou, China
}

Correspondence should be addressed to Chuanbin Wu, cbwu2000@yahoo.com

Received 25 April 2011; Revised 25 June 2011; Accepted 26 June 2011

Academic Editor: Xing J. Liang

Copyright ( 12011 Zhiwen Yang et al. This is an open access article distributed under the Creative Commons Attribution License, which permits unrestricted use, distribution, and reproduction in any medium, provided the original work is properly cited.

In order to develop an oral formation of Amphotericin B (AmB) using phytantriol- (PYT-) based cubosomes with desirable properties, homogenization conditions were firstly investigated to determine their effects on the morphological and dimensional characteristics of cubosomes. Under the optimized homogenization conditions of 1200 bar for 9 cycles, cubosomes with reproducible, narrow particle size distribution and a mean particle size of $256.9 \mathrm{~nm} \pm 4.9 \mathrm{~nm}$ were obtained. The structure of the dispersed cubosomes was revealed by SAXS (small-angle X-ray scattering) and Cryo-TEM (cryogenic transmission electron microscopy) as a bicontinuous cubic liquid crystalline phase with $\mathrm{Pn} 3 \mathrm{~m}$ geometry. To overcome the poor drug solubility and increase the drug-loading rate, a solubilization method was firstly used to develop cubosomes containing AmB. The encapsulation efficiency determined by HPLC assay was $87.8 \% \pm 3.4 \%$, and UV spectroscopy and stability studies in simulated gastric fluids further confirmed that AmB was successfully encapsulated in cubosomes.

\section{Introduction}

Amphotericin B has been widely used in clinics and is still regarded as the golden standard and a life-saving drug in the treatment of severe fungal infections for cancer, organ, or bone marrow transplant patients. Especially important, as one of the oldest antimicrobial antibiotics, AmB has not induced resistance in the clinical treatment. However, clinical applications of AmB are particularly limited because of its parenteral administration and potential organ toxicity, such as nephrotoxicity, central nervous system, and liver damage [1].

An effective and safe oral formulation of $A m B$ will surely have significant applications in the treatment of disseminated fungal infections, because it will eliminate acute toxicity (fever and chilling) associated with intravenous (i.v.) delivery, reduce renal toxicity, shorten or avoid hospitalization, and increase patient satisfaction. Unfortunately, due to its low solubility and poor membrane permeability, oral administration of $\mathrm{AmB}$ was reported to result in a poor bioavailability [2-5]. With the disappointed legacy of clinical trial failure and the physicochemical properties that precluded dissolution, it has been commonly believed that a formulation of $\mathrm{AmB}$ for oral administration is not a viable therapeutic strategy.

In recent years, advances in drug-delivery technologies and in-depth understanding of alternative delivery routes have inspired scientists to reassess AmB for oral administration. As a result, a substantial progress towards oral formulations of $\mathrm{AmB}$ has been achieved, such as $\mathrm{AmB}$ nanosuspension, lipid-based cochleates, and lipid nanospheres [6-8]. More encouragingly, the oral $\mathrm{AmB}$ cochleate formulation is currently under development by Biodelivery Sciences, Inc. (BDSI) and a recent press release regarding the favorable outcome of phase I trial was released in February 2009, and 
the company emphasized that further plans of clinical trials were underway.

Because of their multilayer structures consisting of continuous lipid bilayers which are similar to those of cochleates, cubosomes are considered as novel lipid-based delivery vehicles that have potential for delivery of AmB. Cubosomes are nanoparticles, more accurately nanostructured liquidcrystalline particles $[9,10]$, in a liquid crystalline phase with cubic crystallographic symmetry formed by self-assembly of amphiphilic or surfactant-like molecules. This unique liquid crystalline structure of cubosomes could provide protection for entrapped drug from degradation in the gastrointestinal tract [11-13]. Indeed, the dispersed cubosomes have been highly recommended as carriers for active molecules due to their low viscosity, large interfacial areas, and the presence of both hydrophilic and hydrophobic regions. Furthermore, because of their great biocompatibility and bioadhesivity, cubosomes are suitable for oral administration [13-17]. For example, oral administration of cubosomes loaded with insulin resulted in a sustained reduce of blood glucose levels in rats [14].

Initially, the common method using high-energy dispersion of bulk cubic phase was applied to prepare phytantriolbased cubosomes containing AmB $[18,19]$. However, this method had two major obstacles which made it very difficult to produce dispersed cubosomes with desirable properties, such as uniform particle size distribution and valuable drugloading rate. Firstly, in the previously published studies which prepared cubosomes by homogenizing a certain amount of larger particles at 600 bar [20-22], it was emphasized that the fraction of larger particles in the obtained cubosomes should be taken into account for dispersion characterization as well as in vitro and in vivo studies performed on cubosomes. However, by now, no experiment had been conducted to show how to control the size distribution of dispersed cubosomes via using high-energy dispersion method. It was not clear whether the cubic liquidcrystalline structure of cubosomes was still maintained or disrupted under the high energy homogenization. Secondly, due to its amphiphilic properties, $\mathrm{AmB}$ is poorly soluble in water, oil, and many organic solvents. With a low solubility of AmB in PYT (less than $1 \mathrm{mg} / \mathrm{g}$ ), it seems impossible to develop phytantriol-based cubosomes containing AmB with a valuable drug-loading rate.

In order to overcome these obstacles, different homogenization conditions were investigated in this study to determine the optimal conditions to produce phytantriol-based cubosomes with uniform particle size and distribution. Also, water-soluble $\mathrm{AmB}$ deoxycholate was selected to enhance the solubility of AmB in dispersed cubosomes. More specifically, the aim of this work was to optimize the preparation method for phytantriol-based cubosomes containing AmB with desirable properties.

\section{Materials and Methods}

2.1. Materials. Amphotericin B was obtained from North China Pharmaceutical Group Corporation (Shenyang, China). Phytantriol was purchased from DSM (Basel,
Switzerland). Poloxamer 407 ( $\left.\mathrm{PEO}_{98} \mathrm{POP}_{67} \mathrm{PEO}_{98}\right)$ was a gift from BASF (Ludwigshafen, Germany). Deoxycholate Sodium was purchased from Amresco (USA). Glass capillaries for SAXS experiments were obtained from Anton Paar (Graz, Austria). All the chemicals were used as received without further purification. Milli-Q grade water purified through a Millipore system (ELGA LabWater, Sartorius, UK) was used throughout this study.

\subsection{Preparation of Phytantriol-Based Cubosomes}

2.2.1. Production of Blank Cubosomes. Proper amount of phytantriol, Poloxamer 407 and water were weighed for preparation of bulk cubic phases. Phytantriol and P407 at a ratio of $9: 1(\mathrm{w} / \mathrm{w})$ were melted at $60^{\circ} \mathrm{C}$ in a hot water bath till homogenous. Then, $2 \mathrm{~mL}$ of deionized water were added gradually and vortex mixed for $1 \mathrm{~min}$ to achieve a homogenous state. The samples were equilibrated at room temperature for $48 \mathrm{hrs}$, and then, optically isotropic cubicphase gels were formed. Subsequent fragmentation in $20 \mathrm{~mL}$ of water was performed by intermittent probe sonication (JYD-650, Shanghai, China) for $10 \mathrm{~min}$ using a pulse mode ( $9 \mathrm{sec}$ pulses interrupted by $18 \mathrm{sec}$ breaks) at $400 \mathrm{~W}$ energy input. The resulting milky coarse dispersions were homogenized using a high-pressure homogenizer (Avestin Em-C3, Ottawa, Canada) at certain high pressures and cycles to obtain an opalescent dispersion of the cubosomes. The final dispersions of cubic-phase gel were stored at room temperature for later studies.

2.2.2. Production of Cubosomes Containing AmB. In the case of preparing phytantriol-based cubosomes containing AmB, $\mathrm{AmB}$ deoxycholate (equivalent to $60 \mathrm{mg}$ of $\mathrm{AmB}$ ) was firstly dissolved in $2 \mathrm{~mL}$ of deionized water. Phytantriol and $\mathrm{P} 407$ at a ratio of $9: 1(\mathrm{w} / \mathrm{w})$ were melted at $60^{\circ} \mathrm{C}$ in a hot water bath till homogenous. Then, the AmB deoxycholate solution was added in gradually and vortex mixed for $1 \mathrm{~min}$ to achieve a homogenous state. The remaining process followed the same steps described above for preparation of blank cubosomes.

\subsection{Characterization of Cubosomes}

2.3.1. Particle Size Measurements. The cubosomal particle size was determined by photon correlation spectroscopy using a Zetasizer Nano ZS90 (Malvern Instruments, Malvern, $\mathrm{UK}$ ) at $25^{\circ} \mathrm{C}$, which is based on the principles of Brownian motion. Samples were diluted in particle-free purified water to a scattering intensity of approximately $150-300 \mathrm{kcps}$ and the dispersant viscosity was set to $0.8872 \mathrm{cP}$ at $25^{\circ} \mathrm{C}$. The mean $\mathrm{z}$-average diameter and polydispersity indices (PDI) were obtained by cumulate analysis using the MALVERN software.

2.3.2. Cryogenic Transmission Electron Microscopy (CryoTEM). For cryo-TEM examination, $4 \mu \mathrm{L}$ of cubosome sample were applied on a carbon-coated holey film grid and then gently blotted with filter paper for about 3 seconds to obtain a thin liquid film on the grid. Immediately after blotting, the grid was plunged into precooled liquid ethane for flash 
freeze. Then the cryo-grid was held in a Gatan 626 CryoHolder (Gatan, USA) and the sample was transferred into a cryo-transmission electron microscope (cryo-TEM; JOEL JEM-2010, Tokyo, Japan) at $-172^{\circ}$ C. Samples were observed under minimal dose condition and the images were recorded digitally by a CCD camera (Gatan 832) at the defocus of 3$5.464 \mu \mathrm{m}$.

2.3.3. Small-Angle X-Ray Scattering (SAXS). Small-angle Xray scattering (SAXS) measurements were carried out on a high-flux SAXS instrument (SAXSess, Anton Paar, Graz, Austria) operating in line collimation and equipped with an imaging plate (IP) as a detector. The IP with a pixel size of $42.3 \times 42.3 \mu \mathrm{m}^{2}$ extended into a wide-angle range (the $q$ range covered by the IP was up to $28 \mathrm{~nm}^{-1}, q=$ $(4 \pi \sin \theta) / \lambda$, where $\lambda$ is the wavelength of $0.1542 \mathrm{~nm}$ and $2 \theta$ is the scattering angle). The liquid samples were carefully loaded into a quartz capillary with a diameter of $1 \mathrm{~mm}$. The gel samples were wrapped by aluminum foil and then fastened onto the sample holder. The exposure time was $60 \mathrm{~min}$ for the liquid samples and $20 \mathrm{~min}$ for the gels.

\subsection{Confirmation of Cubosomes Containing AmB}

2.4.1. Encapsulation Efficiency. With the aim to quantify $\mathrm{AmB}$ content encapsulated in cubosomes after production, $0.5 \mathrm{~mL}$ of cubosomes containing $\mathrm{AmB}$ was added into the reservoir of centricon (YM-100, Amicon, Millipore, Bedfore, Mass, USA) [23]. After centrifuging the cubosome dispersion at $15,000 \mathrm{rpm}$ for $30 \mathrm{~min}$, the filtrate which contained free $A m B$ was removed. In the process of centrifuge, free $\mathrm{AmB}$ (water soluble AmB deoxycholate) was removed from cubosomal formulation, because the bottom of the reservoir of centricon has the certain size of holes. Then, the filtered dispersion was diluted with methanol and analyzed for entrapped $\mathrm{AmB}$ content using high performance liquid chromatography (HPLC).

2.4.2. UV Spectroscopic Examination. The spectra of AmB in different samples, which included $A m B$ deoxycholate, cubosomes containing $\mathrm{AmB}$, and a mixture of $\mathrm{AmB}$ deoxycholate and blank cubosomes all at the $\mathrm{AmB}$ concentration of $2 \mathrm{mg} / \mathrm{mL}$, were investigates by a UV-vis spectrometer [24] at the wavelength of $250 \mathrm{~nm}$ to $500 \mathrm{~nm}$. The ratio of the absorbance of the first peak (I) to the last peak (IV) was used to monitor the aggregation state of AmB.

2.4.3. Stability Studies in Simulated Gastric Fluids (SGFs). $\mathrm{AmB}$ deoxycholate, cubosomes containing $\mathrm{AmB}$, and a mixture of $\mathrm{AmB}$ deoxycholate and blank cubosomes all at the $\mathrm{AmB}$ concentration of $2 \mathrm{mg} / \mathrm{mL}$ were placed in SGF at the dilution rate of $1: 10(\mathrm{v} / \mathrm{v})$ at $25^{\circ} \mathrm{C}$. After $120 \mathrm{~min}$ of incubation, the samples were characterized by optical microscopy (Nikon Diaphot, Nikon, Tokyo, Japan). The samples were, respectively, placed on a microscopy glass over the objective of the microscope and stood for equilibration. After 40 to 60 seconds when the small particles slowed down the visible movement, photomicrographs were taken.
TABLE 1: Results of particle size measurement on three batches of blank cubosome dispersions prepared under different homogenization conditions.

\begin{tabular}{lcc}
\hline Cubosome dispersion & Mean particle size $(\mathrm{nm})$ & PDI \\
\hline Ultrasound only & $291.70 \pm 37.40$ & $0.250 \pm 0.030$ \\
600 bar for 3 cycles & $290.00 \pm 25.00$ & $0.210 \pm 0.024$ \\
600 bar for 6 cycles & $277.33 \pm 4.16$ & $0.191 \pm 0.027$ \\
600 bar for 9 cycles & $275.33 \pm 18.82$ & $0.189 \pm 0.009$ \\
1200 bar for 3 cycles & $259.66 \pm 19.30$ & $0.162 \pm 0.010$ \\
1200 bar for 6 cycles & $259.67 \pm 12.42$ & $0.149 \pm 0.027$ \\
1200 bar for 9 cycles & $241.00 \pm 12.42$ & $0.107 \pm 0.027$ \\
1800 bar for 9 cycles & $248.67 \pm 4.93$ & $0.103 \pm 0.016$ \\
\hline
\end{tabular}

\section{Results}

\subsection{Optimizing the Preparation Process for PYT-Based Cubosomes Containing AmB}

3.1.1. Effects of Homogenization Conditions on Particle Size of Cubosomes. Previously, it was reported that the produced cubosomes always contained a certain amount of large particles [20-22]. In order to obtain PYT-based cubosomes with fewer or no large irregular particles, this study investigated the influence of experimental parameters, specifically the homogenization pressures and the number of homogenization cycles, on the morphological and dimensional characteristics of the produced cubosomes.

Cubosomes were produced with phytantriol/Poloxamer 407 ratio of $9: 1 \mathrm{w} / \mathrm{w}$ in water and dispersed phase/dispersing phase ratio of 5:95. The mean diameter of different cubosome dispersions was in an approximate range of 241$291 \mathrm{~nm}$ with the polydispersity indices (PDI) value of 0.25 for sonicated cubosomes and below 0.21 for homogenized cubosomes. After homogenization at 600 bar for 9 cycles, the particle size of cubosomes was reduced from micrometer to nanometer ranges. As shown in Figure 1, a few particles larger than $1 \mu \mathrm{m}$ were still found in cubosome dispersion which was homogenized at 1200 bar for 6 cycles. With increasing the homogenization cycles to 9 at 1200 bar, cubosomes with reproducible particle size and size distribution were obtained. Further increasing the homogenization pressure to 1800 bar for 9 cycles did not show significant effect on reducing particle size and narrowing size distribution.

Simultaneously, the influence of homogenization conditions on the particle size of PYT-based cubosomes containing $\mathrm{AmB}$ were also investigated, and similar results as those for blank cubosomes were obtained (data not shown). Based on the results of particle size measurements, it can be recommended that phytantriol-based cubosomes with nanometer-sized particles, reproducible and narrow particle size distribution could be produced under the optimized homogenization conditions of 1200 bar and 9 cycles (Table 1).

3.1.2. Effect of AmB Content on Encapsulation Efficiency. The encapsulation efficiency of phytantriol-based cubosomes containing $\mathrm{AmB}$ was evaluated by adding different amount 


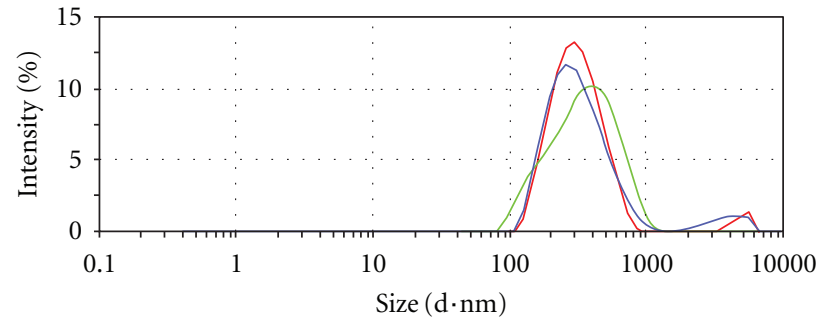

(a)

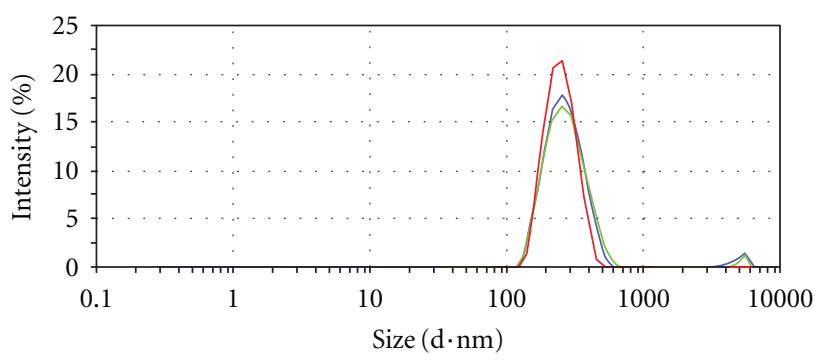

(c)

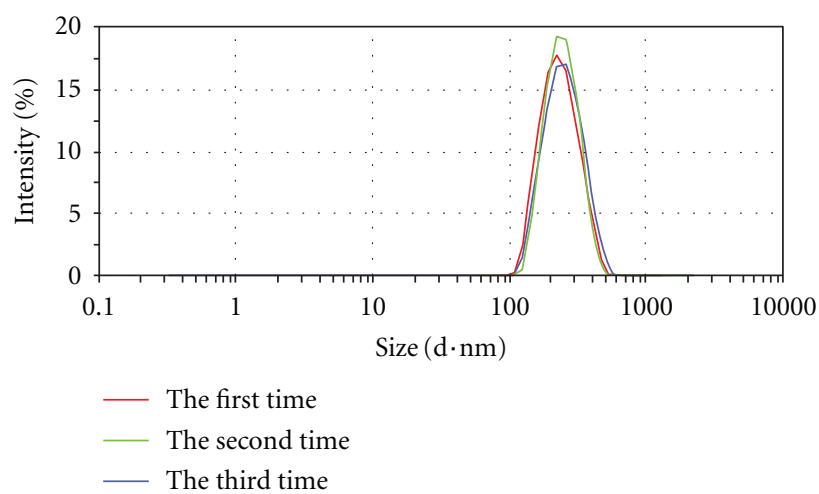

(e)

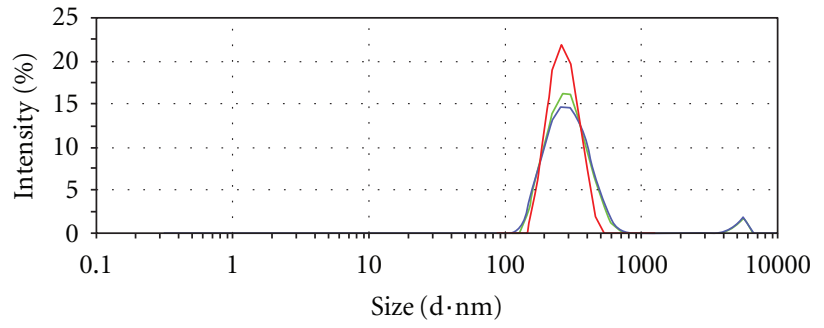

(b)

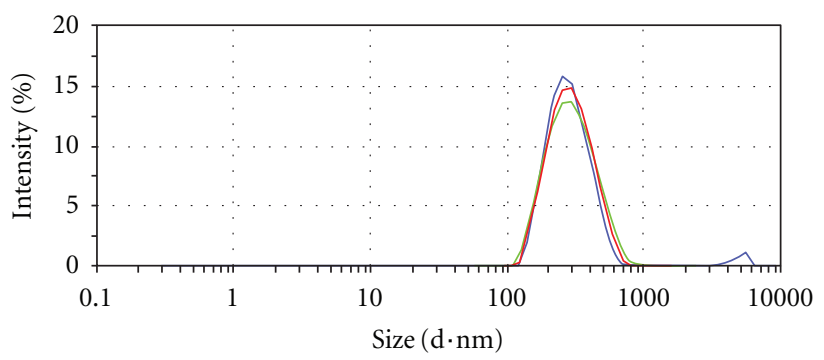

(d)

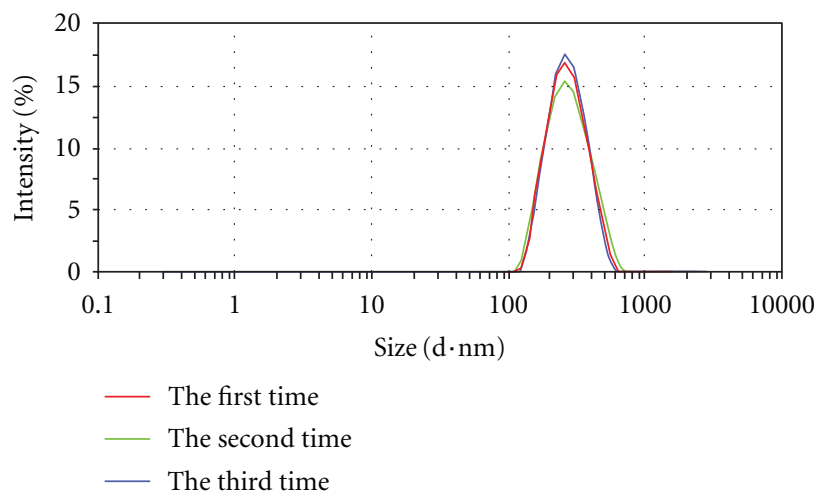

(f)

FIgURE 1: Particle size distribution of cubosome dispersion measured for three times via Zetasizer Nano ZS90. Homogenization conditions: (a) ultrasound only; (b) 600 bar for 3 cycles; (c) 600 bar for 9 cycles; (d) 1200 bar for 6 cycles; (e) 1200 bar for 9 cycles; (f) 1800 bar for 9 cycles.

of $\mathrm{AmB}$ (30 mg, $45 \mathrm{mg}$, and $60 \mathrm{mg}$ ) to the dispersed phase during preparation. The results in Table 2 showed that $A m B$ encapsulation efficiency was $88.5 \% \pm 0.6 \%, 91.8 \% \pm 1.5 \%$, and $87.8 \% \pm 3.4 \%$, respectively, and no significant effect of added $\mathrm{AmB}$ content on encapsulation efficiency was found. Therefore, based on the maximum solubility of AmB (60 mg of AmB dissolved in $2 \mathrm{~mL}$ of deionized water), PYT-based cubosomes containing $60 \mathrm{mg} \mathrm{AmB}$ with the highest drugloading rate of $3.0 \%$ were chosen for the following studies.

\subsubsection{Effect of AmB Content on Particle Size of Cubosomes.} The influence of $\mathrm{AmB}$ content added to the dispersed phase during preparation on the particle size of PYTbased cubosome dispersions was investigated by applying homogenization at 1200 bar for 9 cycles. The results in Table 3 indicated that there was no significant difference in
TABLE 2: Effect of AmB content on encapsulation efficiency $(n=3)$.

\begin{tabular}{lcc}
\hline $\begin{array}{l}\text { Amount of AmB }(\mathrm{mg}) \\
\text { added to cubosomes }\end{array}$ & $\begin{array}{c}\text { Encapsulation } \\
\text { efficiency }(\%)\end{array}$ & $\begin{array}{c}\text { Actual drug-loading } \\
\text { rate }(\%)\end{array}$ \\
\hline 30 & $88.5 \pm 0.6$ & 1.5 \\
45 & $91.8 \pm 1.5$ & 2.2 \\
60 & $87.8 \pm 3.4$ & 3.0 \\
\hline
\end{tabular}

particle size of cubosome dispersions with increasing the content of $\mathrm{AmB}$ from $30 \mathrm{mg}$ to $60 \mathrm{mg}$.

\subsection{Characterization of Cubosomal Structure}

3.2.1. SAXS. The cubic phase can be fragmented into stable submicron sized particles, also known as cubosomes, which 
TABle 3: Effect of AmB content on particle size of cubosome dispersions $(n=3)$.

\begin{tabular}{lcc}
\hline $\begin{array}{l}\text { Amount of AmB }(\mathrm{mg}) \\
\text { added to cubosomes }\end{array}$ & Mean particle size $(\mathrm{nm})$ & PDI \\
\hline 30 & $266.0 \pm 3.2$ & $0.12 \pm 0.02$ \\
45 & $259.7 \pm 1.9$ & $0.09 \pm 0.02$ \\
60 & $256.9 \pm 4.9$ & $0.10 \pm 0.04$ \\
\hline
\end{tabular}

retain the internal structure of the original liquid crystalline. Cubosome formation and structure were primarily characterized by cryo-TEM in combination with SAXS to confirm the internal nanostructure of the particles. The homogenized dispersions were analyzed by X-ray diffraction and cryo-TEM, and the results indicated that the cubic structure was remained in cubosomes. Although the dispersion homogenized at 1800 bar for 9 cycles presented the same characteristics as dispersion homogenized at 1200 bar for 9 cycles, the greater shear forces applied might break down the cubic structural particles into nonequilibrium vesicular structures which later would typically fuse into unstable colloidal larger particles. Therefore, the dispersions homogenized at 1200 bar for 9 cycles were subjected to the later studies as drug carrier for oral administration.

Figure 2(d) showed the SAXS profiles of the bulk cubic phase with melts of phytantriol and Poloxamer 407 at a ratio of $9: 1$. Diffraction peaks were observed up to the sixth order at ratios of $2^{1 / 2}: 3^{1 / 2}: 4^{1 / 2}: 6^{1 / 2}: 8^{1 / 2}: 9^{1 / 2}$, which were indicated as the Miller indices, $h k l=110,111,200$, 211,220 , and 221 correspondingly. The observation fits the characteristic peaks of Pn3m crystallographic space group (Q224) which contains a diamond cube phase, and it is also in agreement with the previously published phase behavior of phytantriol [25].

Figure 2(a) showed the SAXS profiles obtained from the phytantriol-based cubosome dispersion homogenized at 1200 bar for 9 cycles. Comparing with the SAXS profiles of bulk cubic phase in Figure 2(d), five similar reflections following the relationship of $2^{1 / 2}, 3^{1 / 2}, 4^{1 / 2}, 6^{1 / 2}$, and $8^{1 / 2}$, which were indexed as $h k l=110,111,200,211$, and 220 peaks of a primitive cubic lattice of the $\mathrm{Pn} 3 \mathrm{~m}$ space group, were observed for cubosome dispersion. Meantime, the same peak positions for these five reflections were detected for cubosome dispersion homogenized at 1800 bar for 9 cycles as illustrated in Figure 2(b). This confirmed that the internal nanostructure of these two cubosome dispersions was Pn $3 \mathrm{~m}$ space group; in other words, the particles kept the bicontinuous cubic nature after homogenization. However, the weak peaks of 200,211, and 220 might be attributed to the homogenization destroy.

Furthermore, Figure 2(c) showed the SAXS profiles obtained from the phytantriol-based cubosomes containing $60 \mathrm{mg}$ of AmB homogenized at 1200 bar for 9 cycles. In this case, only three reflections following the relationship of $2^{1 / 2}$, $3^{1 / 2}$, and $4^{1 / 2}$, which were indexed as $h k l=110,111$, and 200 peaks of a primitive cubic lattice of the Pn $3 \mathrm{~m}$ space group, were observed. The disappearing of other weak peaks is more likely due to the fact that some drug molecules attached to the surface of cubosomes and thus interfered with the diffraction peaks, rather than destroying the cubic liquidcrystalline structure by adding the drug. The direct evidence came from cryo-TEM below, in which the cubosomal characterization was obvious in PYT-based cubosomes containing AmB. Thus, based on the SAXS results, the internal structure of phytantriol-based cubosomes containing $60 \mathrm{mg} \mathrm{AmB}$ was the Pn3m crystallographic space group (Q224).

3.2.2. Cryo-TEM. In order to further investigate the cubic structure of the blank phytantriol-based cubosome dispersions and cubosomes containing $\mathrm{AmB}$ homogenized at 1200 bar for 9 cycles, the formulations were examined by cryoTEM. Figure 3(a) showed the image of blank cubosomes which contained highly ordered cubic inner structure with a mean particle size of about $250 \mathrm{~nm}$ close to that observed by laser diffraction. Figure 3(b) showed the image of cubosomes containing $\mathrm{AmB}$ with typical cubosomal characterization and a mean particle size of about $250 \mathrm{~nm}$ similar to that of blank cubosomes. The typical crystal structure obtained via lowering the brightness of cryo-TEM was observed to confirm the cubic liquid-crystalline structure of AmB-loaded cubosomes, as seen in the upper right corner of Figure 3(b).

\subsection{Characterization of Phytantriol-Based Cubosomes Containing AmB}

3.3.1. Encapsulation Efficiency. As mentioned before, the encapsulation efficiency of phytantriol-based cubosomes containing $\mathrm{AmB}$ was measured by removing any free $\mathrm{AmB}$ through filtration and only detecting the entrapped AmB. The results in Table 2 demonstrated that as much as $90 \%$ $\mathrm{AmB}$ was successfully encapsulated in phytantriol-based cubosomes.

3.3.2. UV Spectroscopy. The UV spectrum of AmB depends on its aggregation state. Generally, in aqueous solution, $\mathrm{AmB}$ is aggregated, and its absorption spectrum presents a major broad band at $328 \mathrm{~nm}$. In this study, the absorption spectra of AmB in four samples, including AmB deoxycholate, cubosomes containing $\mathrm{AmB}$, and a mixture of $\mathrm{AmB}$ deoxycholate and blank cubosomes which all contained $2 \mathrm{mg} / \mathrm{mL}$ of $\mathrm{AmB}$ as well as blank cubosomes, were compared to demonstrate the successful encapsulation of $\mathrm{AmB}$ in cubosomes. As shown in Figure 4, the major absorption band was observed in both free AmB solution and physical mixture of $A m B$ and cubosomes. The spectroscopy of cubosomes containing $\mathrm{AmB}$ showed the absorption peak at different position with much lower intensity, which suggested that $\mathrm{AmB}$ was actually encapsulated in phytantriol-based cubosomes rather than simply mixed with blank cubosomes.

3.3.3. Stability Studies in Simulated Gastric Fluids. Stability studies were performed on AmB deoxycholate, cubosomes containing $\mathrm{AmB}$, and a mixture of $\mathrm{AmB}$ deoxycholate and blank cubosomes which all contained $2 \mathrm{mg} / \mathrm{mL}$ of AmB. After incubation for $120 \mathrm{~min}$, some changes were optically shown in Figure 5. AmB aggregation was found in 

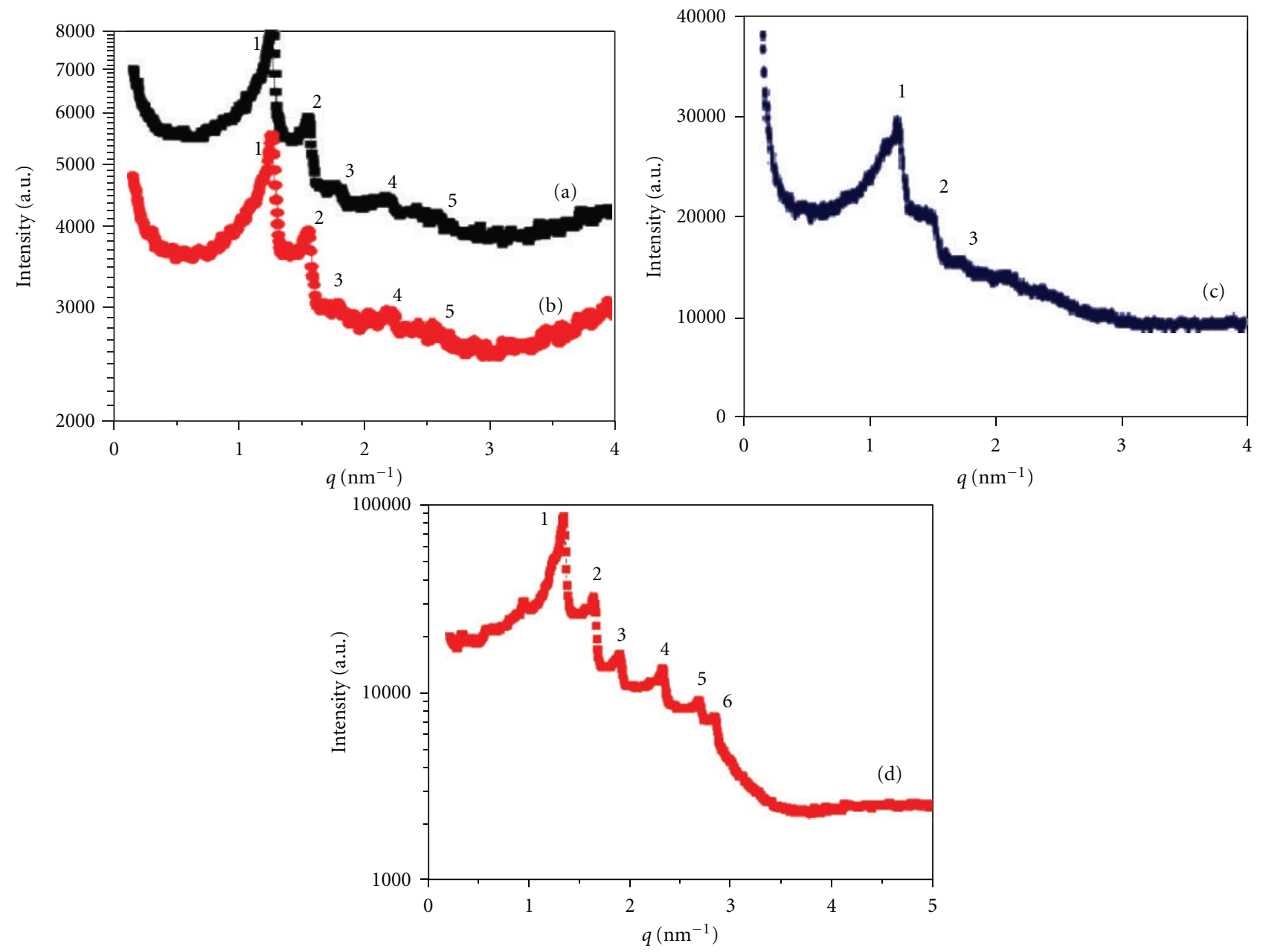

FIgURE 2: SAXS profiles of the cubic liquid-crystalline structure: (a) phytantriol-based cubosome dispersions homogenized at 1200 bar for 9 cycles; (b) at 1800 bar for 9 cycles; (c) phytantriol-based cubosomes containing AmB homogenized at 1200 bar for 9 cycles; (d) the bulk cubic phase with melts of phytantriol and Poloxamer 407 at a ratio of $9: 1$.

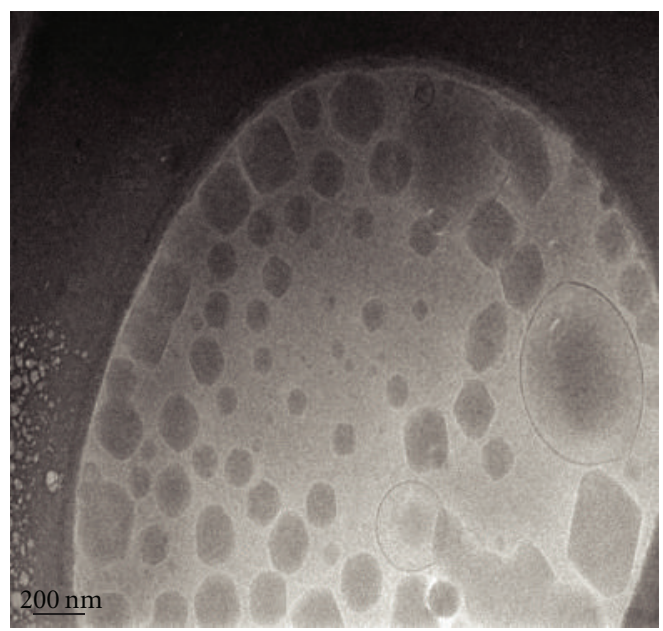

(a)

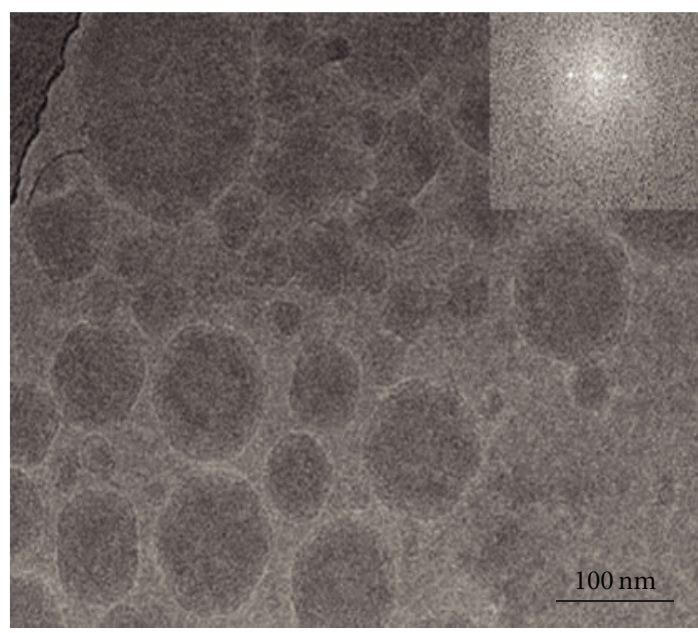

(b)

Figure 3: Cryo-TEM micrographs of blank phytantriol-based cubosomes (a) and cubosomes containing AmB (b). 


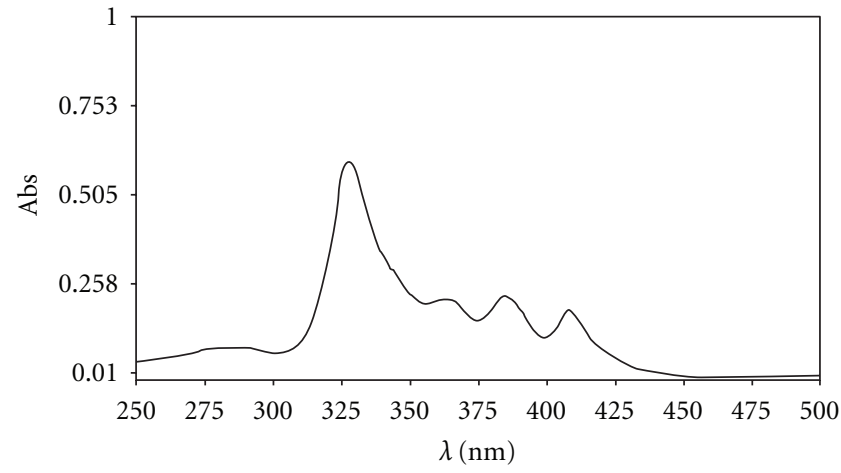

(a)

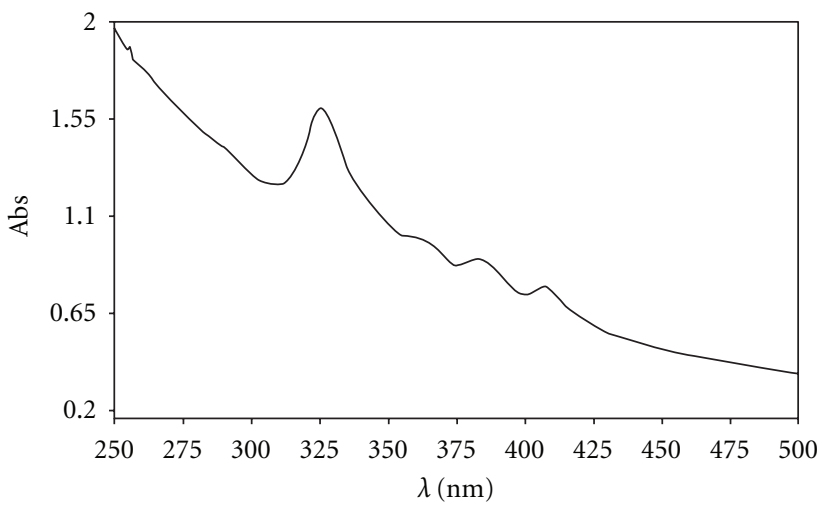

(c)

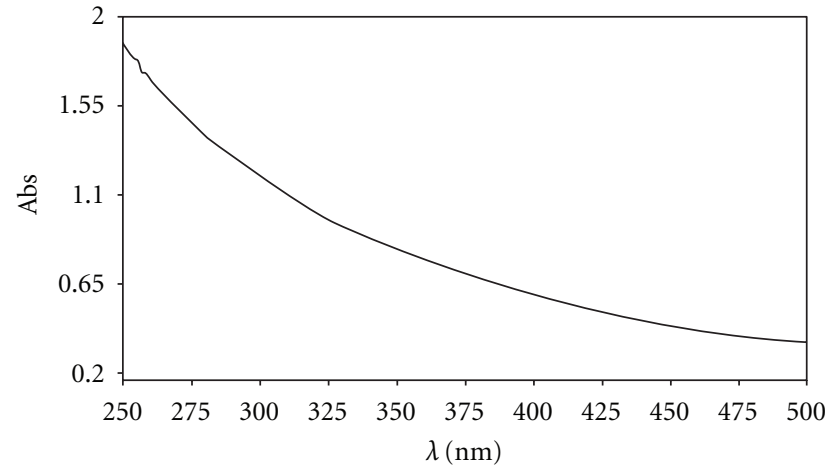

(b)

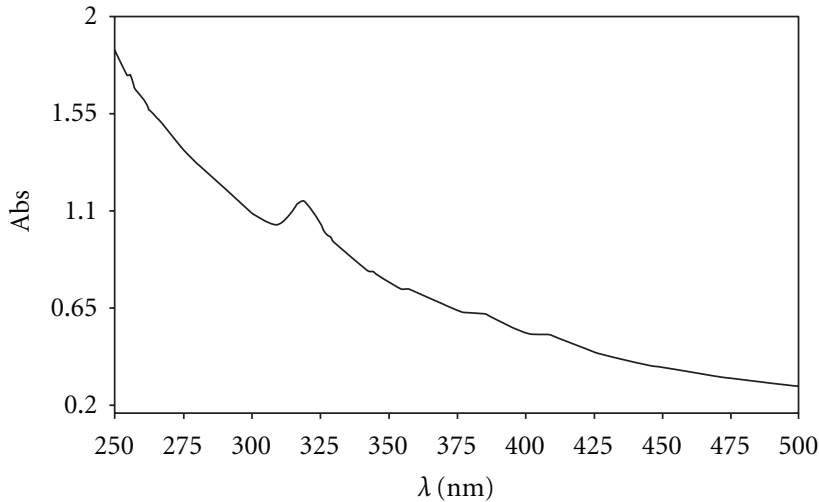

(d)

Figure 4: UV spectra of AmB. (a) AmB deoxycholate, (b) blank cubosomes, (c) mixture of AmB deoxycholate and blank cubosomes, and (d) cubosomes containing AmB.

both $\mathrm{AmB}$ solution and mixture of $\mathrm{AmB}$ and cubosomes (Figures 5(a) and 5(b)), while dispersed AmB was clearly observed in cubosomes containing AmB (Figure 5(c)). The different states of $\mathrm{AmB}$ observed in cubosomes containing $\mathrm{AmB}$ and the mixture of $\mathrm{AmB}$ and blank cubosomes demonstrated that AmB was successfully encapsulated in PYT-based cubosomes rather than simply mixed with the cubosomes. Optical observation used to evaluate the cubosomes stability is that $\mathrm{AmB}$ aggregation was easy happened to become a large aggregation size, which could not be determined by photon correlation spectroscopy (PCS).

\section{Discussion}

The advantages of cubosomes as oral delivery system may lie in the following aspects. Firstly, the lyotropic property of cubosomes with hydrophilic surface makes the contact with the endothelial cell membrane more easily and overcomes the "unstirred water layer" barrier [26-28]. Secondly, either the cubic nanoparticles or the encapsulated drug molecules can enhance the penetration across the endothelial cell membrane, which will increase the uptake efficiency of orally administrated drug [29]. Currently, in the majority of reported studies on dispersed liquid crystalline systems, glyceryl monoolein (GMO) was used for preparation [17] as it could form a bicontinuous cubic phase in excess water. However, GMO-based cubosomes consisted of lipid composition have the same digestion process in the gastrointestinal tract as micelles, liposomes, and niosomes because the ester-based structure may be rapidly cleaved by pancreatic lipase, and the ester hydrolysis is likely to induce chemical instability and disruption of the liquid crystalline structure during the digestion. To avoid this shortcoming, one alternative resolution is PYT-based cubosomes, because phytantriol does not possess a susceptible ester bond in its molecular structure, and it is likely the cubic phase structure can be retained under the digestive conditions. Therefore, phytantriol-based cubosomes were firstly investigated as drug-delivery system in the present study.

First of all, the optimal formulation process for phytantriol-based cubosomes containing $\mathrm{AmB}$ was determined in order to produce cubosomes with desirable particle size and distribution and good drug-loading rate as oral drug delivery carriers. Cubosomal size is an important parameter, which could influence drug release, intestinal uptake, and thus the overall bioavailability of the formulation $[30,31]$. The particles in a size of $100 \mathrm{~nm}$ generally present a significantly higher uptake by the intestinal tissues in terms of total mass and quantity compared to the larger particles. This is because of a particle size-dependent exclusion phenomena in which smaller particles are more likely to be internalized inside the cells and tissues [31, 32]. Unfortunately, PYT-based 


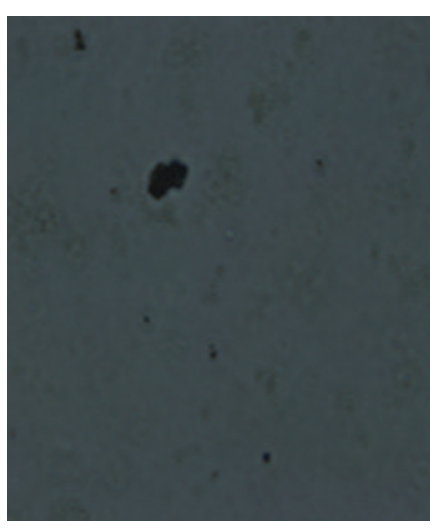

(a)

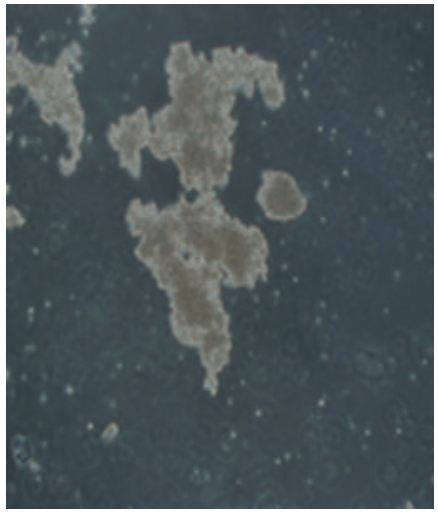

(b)

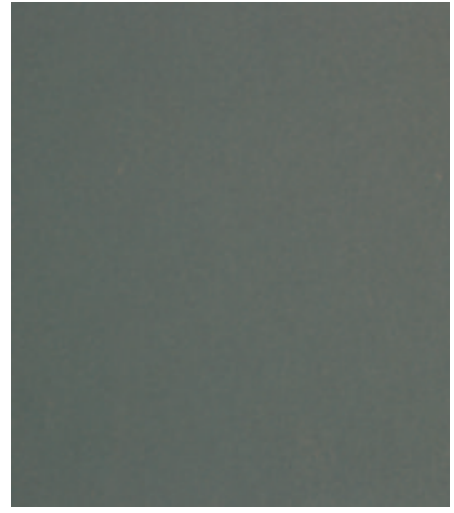

(c)

Figure 5: Optical microscopy of samples in SGF $(\times 200)$. (a) AmB deoxycholate, (b) mixture of AmB deoxycholate and blank cubosomes, and (c) cubosomes containing AmB.

cubosomes with a mean particle size of $250 \mathrm{~nm}$ and uniform size distributions were only obtained in this study.

To date, the poor solubility of $\mathrm{AmB}$ in oil is still a challenge, and no effective solutions have been found to enhance the solubility. Recently, a self-emulsifying drugdelivery system (SEDDS) using lipidic excipient Peceol was reported to improve the oral absorption of $\mathrm{AmB}$ [33]. However, solubilization of AmB in SEDDS formulations was limited at $100-500 \mu \mathrm{g} / \mathrm{mL}$, which led to an unacceptable amount of lipid to be administered. With AmB solubility in PYT less than $1 \mathrm{mg} / \mathrm{g}$, it seems also impracticable to develop phytantriol-based cubosomes containing $\mathrm{AmB}$ due to the low drug-loading rate. To increase drug content, several ways have been tested. Firstly, the concentration of PYT was raised to $10 \% \mathrm{wt} / \mathrm{wt}$, but the higher concentration of dispersed phase led to formation of larger particles and a number of aggregates. Secondly, it was reported previously the use of surfactants as solubilizer was able to increase the solubility of AmB. Surfactants, such as F-68, tween-80, sodium dodecylsulfate and sodium deoxycholate, were used in the present study to enhance AmB solubility. But AmB was still not incorporated in cubosomal dispersions. Finally, an efficient method was found to improve the AmB solubility by gradually adding $2 \mathrm{~mL}$ of $\mathrm{AmB}$ deoxycholate solution (maximally $60 \mathrm{mg} \mathrm{AmB}$ was dissolved in $2 \mathrm{~mL}$ of water) into homogeneous melt of Phytantriol and P407. With this method, phytantriol-based cubosomes containing AmB with drug loading rate up to $3 \%$ were successfully produced.

As reported previously, without the aid of an emulsifier, PYT itself did not form a stable emulsion in water [22]. The surfactant is not merely absorbed at the particle surface; actually, the surfactant is more likely anchored at the apolar region or at the surface of PYT-based bilayers [22]. If AmB deoxycholate was melted together with Phytantriol and P407 at $60^{\circ} \mathrm{C}$, deoxycholate might rapidly interact with PYT and destroy the micellar structure of $\mathrm{AmB}$ deoxycholate and accordingly lower the solubilization of AmB then formed $\mathrm{AmB}$ crystals precipitation. By selecting the proper surfactant and controlling the adding time of $\mathrm{AmB}$ deoxycholate, the addition of water soluble AmB deoxycholate into the melt of phytantriol and P407 could keep a enough little drug-particle size for the drug-delivery system subjected to a high-pressure process leading to the complete dissolution of $\mathrm{AmB}$ in the cubosomes.

Theoretically, the AmB could spontaneously diffuse from the aqueous phase into the PYT layer according to its partitioning coefficient. But the possible problem lies in the dissolution of $\mathrm{AmB}$, because poorly soluble drugs show simultaneously a very low-dissolution velocity. Based on this fact, localization of the AmB in the PYT layer appeared feasible to achieve when accelerating the dissolution velocity of AmB. As shown in Noyes-Whitney equation, the higher the streaming velocity of the dissolution medium across the crystal surface is, the larger is the dissolution velocity. Previous research had indicated that the stirring velocities and related streaming velocity of the fluid are enough for some little AmB crystals dissolved in lipid material when homogenizer was employed [34]. This is a reasonable principle for $\mathrm{AmB}$ deoxycholate encapsulated in cubosomes using high pressure homogenization rather than $\mathrm{AmB}$ crystals simply added into cubosomes.

In this study, the results of photon correlation spectroscopy, X-ray diffraction, and cryo-TEM illustrated the cubic nanoparticle structure in the cubosome formulation; however, it was not clarified whether the formulation was a simple mixture of AmB deoxycholate and blank cubosomes or AmB was encapsulated in cubosomes. Therefore, evaluation of encapsulation efficiency, UV spectroscopy measurement, and stability studies in simulated gastric fluids were conducted to confirm the incorporation of drug in cubosomal dispersions. At first, the encapsulated drug content was accurately determined after removing the free drug from the carrying system via ultrafiltration method. The detected encapsulation efficiency of $87.8 \%$ indicated that majority of the drug was indeed encapsulated in cubosomes. Additionally, the comparison of UV spectroscopy demonstrated that when the drug was incorporated in formulation, its absorption peak position and intensity were dramatically influenced. Moreover, the optical microscopy showed the aggregation of AmB in simulated gastric fluids 
during stability study, but the aggregation was replaced by dispersed state of $\mathrm{AmB}$ in cubosomes containing $\mathrm{AmB}$, which also indicated the drug was incorporated in cubosomes. Overall, the characterization results demonstrated the successful preparation of phytantriol-based cubosomes containing $\mathrm{AmB}$ with desirable particle size distribution and valuable drug loading rate. It is necessary to further investigate the cubosomes containing $\mathrm{AmB}$ in vivo and in vitro.

\section{Conclusion}

Phytantriol-based cubosomes containing Amphotericin B as a formulation for oral delivery of $\mathrm{AmB}$, was successfully developed through homogenizing the bulk cubic phase gel into a cubic dispersion. Cubosomes with nanometer-sized particles and reproducible, narrow particle size distribution were optimally obtained under homogenization at 1200 bar for 9 cycles. The structure of the dispersed formulation was confirmed by SAXS and cryo-TEM as a bicontinuous cubic liquid crystalline phase with Pn3m geometry. Encapsulation efficiency, UV spectroscopy, stability studies in simulated gastric fluids further confirmed the incorporation of $\mathrm{AmB}$ in cubosomal dispersions. The cubosomes will be utilized in subsequent studies to determine the oral adsorption of cubosomes as a means to control loading and release of AmB.

\section{Acknowledgments}

The authors are grateful to BASF Company, Germany, for generous gift samples of Poloxamer 407. They thank National Basic Research Program of China (no. 81001643) for the financial support. This work was also supported in part by the Ministry of Science and Technology of Dongguan (Grant no. 2008108101064).

\section{References}

[1] L. Ostrosky-Zeichner, K. A. Marr, J. H. Rex, and S. H. Cohen, "Amphotericin B: time for a new "gold standard"', Clinical Infectious Diseases, vol. 37, no. 3, pp. 415-425, 2003.

[2] J. S. Dangi, S. P. Vyas, and V. K. Dixit, "Effect of various lipid-bile salt mixed micelles on the intestinal absorption of amphotericin-B in rat," Drug Development and Industrial Pharmacy, vol. 24, no. 7, pp. 631-635, 1998.

[3] M. L. Littman, P. L. Horowitz, and J. G. Swadey, "Coccidioidomycosis and its treatment with amphotericin B," The American Journal of Medicine, vol. 24, no. 4, pp. 568-592, 1958.

[4] H. M. Kravetz, V. T. Andriole, M. A. Huber, and J. P. Utz, "Oral administration of solubilized amphotericin B," The New England Journal of Medicine, vol. 265, pp. 183-184, 1961.

[5] D. B. Louria, "Some aspects of the absorption, distribution, and excretion of amphotericin B in man," Antibiotic Medicine \& Clinical Therapy, vol. 5, no. 5, pp. 295-301, 1958.

[6] O. Kayser, C. Olbrich, V. Yardley, A. F. Kiderlen, and S. L. Croft, "Formulation of amphotericin B as nanosuspension for oral administration," International Journal of Pharmaceutics, vol. 254, no. 1, pp. 73-75, 2003.

[7] G. Delmas, S. Park, Z. W. Chen et al., "Efficacy of orally delivered cochleates containing amphotericin B in a murine model of aspergillosis," Antimicrobial Agents and Chemotherapy, vol. 46, no. 8, pp. 2704-2707, 2002.

[8] B. Amarji, Ajazuddin, D. Raghuwanshi, S. P. Vyas, and P. Kanaujia, "Lipid nano spheres (LNSs) for enhanced oral bioavailability of amphotericin B: development and characterization," Journal of Biomedical Nanotechnology, vol. 3, no. 3, pp. 264-269, 2007.

[9] M. L. Lynch, A. Ofori-Boateng, A. Hippe, K. Kochvar, and P. T. Spicer, "Enhanced loading of water-soluble actives into bicontinuous cubic phase liquid crystals using cationic surfactants," Journal of Colloid and Interface Science, vol. 260, no. 2, pp. 404413, 2003.

[10] X. Peng, X. Wen, X. Pan, R. Wang, B. Chen, and C. Wu, "Design and in vitro evaluation of capsaicin transdermal controlled release cubic phase gels," The American Association of Pharmaceutical Scientists Tech, vol. 11, no. 3, pp. 1405-1410, 2010.

[11] C. M. Chang and R. Bodmeier, "Swelling of and drug release from monoglyceride-based drug delivery systems," Journal of Pharmaceutical Sciences, vol. 86, no. 6, pp. 747-752, 1997.

[12] C. M. Chang and R. Bodmeier, "Effect of dissolution media and additives on the drug release from cubic phase delivery systems," Journal of Controlled Release, vol. 46, no. 3, pp. 215222, 1997.

[13] B. Siekmann, H. Bunjes, M. H. J. Koch, and K. Westesen, "Preparation and structural investigations of colloidal dispersions prepared from cubic monoglyceride-water phases," International Journal of Pharmaceutics, vol. 244, no. 1-2, pp. 33-43, 2002.

[14] H. Chung, J. Kim, J. Y. Um, I. C. Kwon, and S. Y. Jeong, "Self-assembled "nanocubicle" as a carrier for peroral insulin delivery," Diabetologia, vol. 45, no. 3, pp. 448-451, 2002.

[15] J. Bender, M. B. Ericson, N. Merclin et al., "Lipid cubic phases for improved topical drug delivery in photodynamic therapy," Journal of Controlled Release, vol. 106, no. 3, pp. 350-360, 2005.

[16] L. B. Lopes, J. L. C. Lopes, D. C. R. Oliveira et al., "Liquid crystalline phases of monoolein and water for topical delivery of cyclosporin A: characterization and study of in vitro and in vivo delivery," European Journal of Pharmaceutics and Biopharmaceutics, vol. 63, no. 2, pp. 146-155, 2006.

[17] B. J. Boyd, D. V. Whittaker, S. M. Khoo, and G. Davey, "Lyotropic liquid crystalline phases formed from glycerate surfactants as sustained release drug delivery systems," International Journal of Pharmaceutics, vol. 309, no. 1-2, pp. 218226, 2006.

[18] Y. D. Dong, I. Larson, T. Hanley, and B. J. Boyd, "Bulk and dispersed aqueous phase behavior of phytantriol: effect of vitamin E acetate and F127 polymer on liquid crystal nanostructure," Langmuir, vol. 22, no. 23, pp. 9512-9518, 2006.

[19] Y. D. Dong, A. W. Dong, I. Larson et al., "Impurities in commercial phytantriol significantly alter its lyotropic liquidcrystalline phase behavior," Langmuir, vol. 24, no. 13, pp. 6998-7003, 2008.

[20] E. Esposito, N. Eblovi, S. Rasi et al., "Lipid-based supramolecular systems for topical application: a preformulatory study.", The American Association of Pharmaceutical Scientists, vol. 5, no. 4 article E30, 2003.

[21] E. Esposito, R. Cortesi, M. Drechsler et al., "Cubosome dispersions as delivery systems for percutaneous administration of indomethacin," Pharmaceutical Research, vol. 22, no. 12, pp. 2163-2173, 2005.

[22] B. Siekmann, H. Bunjes, M. H. J. Koch, and K. Westesen, "Preparation and structural investigations of colloidal dispersions prepared from cubic monoglyceride-water phases," 
International Journal of Pharmaceutics, vol. 244, no. 1-2, pp. 33-43, 2002.

[23] H. Chung, J. Kim, J. Y. Um, I. C. Kwon, and S. Y. Jeong, "Self-assembled "nanocubicle" as a carrier for peroral insulin delivery," Diabetologia, vol. 45, no. 3, pp. 448-451, 2002.

[24] M. L. Adams and G. S. Kwon, "Relative aggregation state and hemolytic activity of amphotericin B encapsulated by poly(ethylene oxide)-block-poly(N-hexyl-L-aspartamide)acyl conjugate micelles: effects of acyl chain length," Journal of Controlled Release, vol. 87, no. 1-3, pp. 23-32, 2003.

[25] F. Muller, A. Salonen, and O. Glatter, "Phase behavior of Phytantriol/water bicontinuous cubic Pn $3 \mathrm{~m}$ cubosomes stabilized by Laponite disc-like particles," Journal of Colloid and Interface Science, vol. 342, no. 2, pp. 392-398, 2010.

[26] T. Korjamo, A. T. Heikkinen, and J. Mönkkönen, "Analysis of unstirred water layer in in vitro permeability experiments," Journal of Pharmaceutical Sciences, vol. 98, no. 12, pp. 44694479, 2009.

[27] A. S. Sallam, E. Khalil, H. Ibrahim, and I. Freij, "Formulation of an oral dosage form utilizing the properties of cubic liquid crystalline phases of glyceryl monooleate," European Journal of Pharmaceutics and Biopharmaceutics, vol. 53, no. 3, pp. 343352, 2002.

[28] J. Y. Um, H. Chung, K. S. Kim, I. C. Kwon, and S. Y. Jeong, "In vitro cellular interaction and absorption of dispersed cubic particles," International Journal of Pharmaceutics, vol. 253, no. 1-2, pp. 71-80, 2003.

[29] K. Katneni, S. A. Charman, and C. J. H. Porter, "An evaluation of the relative roles of the unstirred water layer and receptor sink in limiting the in-vitro intestinal permeability of drug compounds of varying lipophilicity," Journal of Pharmacy and Pharmacology, vol. 60, no. 10, pp. 1311-1319, 2008.

[30] G. Mittal, D. K. Sahana, V. Bhardwaj, and M. N. V. Ravi Kumar, "Estradiol loaded PLGA nanoparticles for oral administration: effect of polymer molecular weight and copolymer composition on release behavior in vitro and in vivo," Journal of Controlled Release, vol. 119, no. 1, pp. 77-85, 2007.

[31] M. P. Desai, V. Labhasetwar, G. L. Amidon, and R. J. Levy, "Gastrointestinal uptake of biodegradable microparticles: effect of particle size," Pharmaceutical Research, vol. 13, no. 12, pp. 1838-1845, 1996.

[32] M. P. Desai, V. Labhasetwar, E. Walter, R. J. Levy, and G. L. Amidon, "The mechanism of uptake of biodegradable microparticles in Caco-2 cells is size dependent," Pharmaceutical Research, vol. 14, no. 11, pp. 1568-1573, 1997.

[33] E. K. Wasan, K. Bartlett, P. Gershkovich et al., "Development and characterization of oral lipid-based Amphotericin B formulations with enhanced drug solubility, stability and antifungal activity in rats infected with Aspergillus fumigatus or Candida albicans," International Journal of Pharmaceutics, vol. 372, no. 1-2, pp. 76-84, 2009.

[34] R. H. Müller, S. Schmidt, I. Buttle, A. Akkar, J. Schmitt, and S. Brömer, "SolEmuls-novel technology for the formulation of i.v. emulsions with poorly soluble drugs," International Journal of Pharmaceutics, vol. 269, no. 2, pp. 293-302, 2004. 

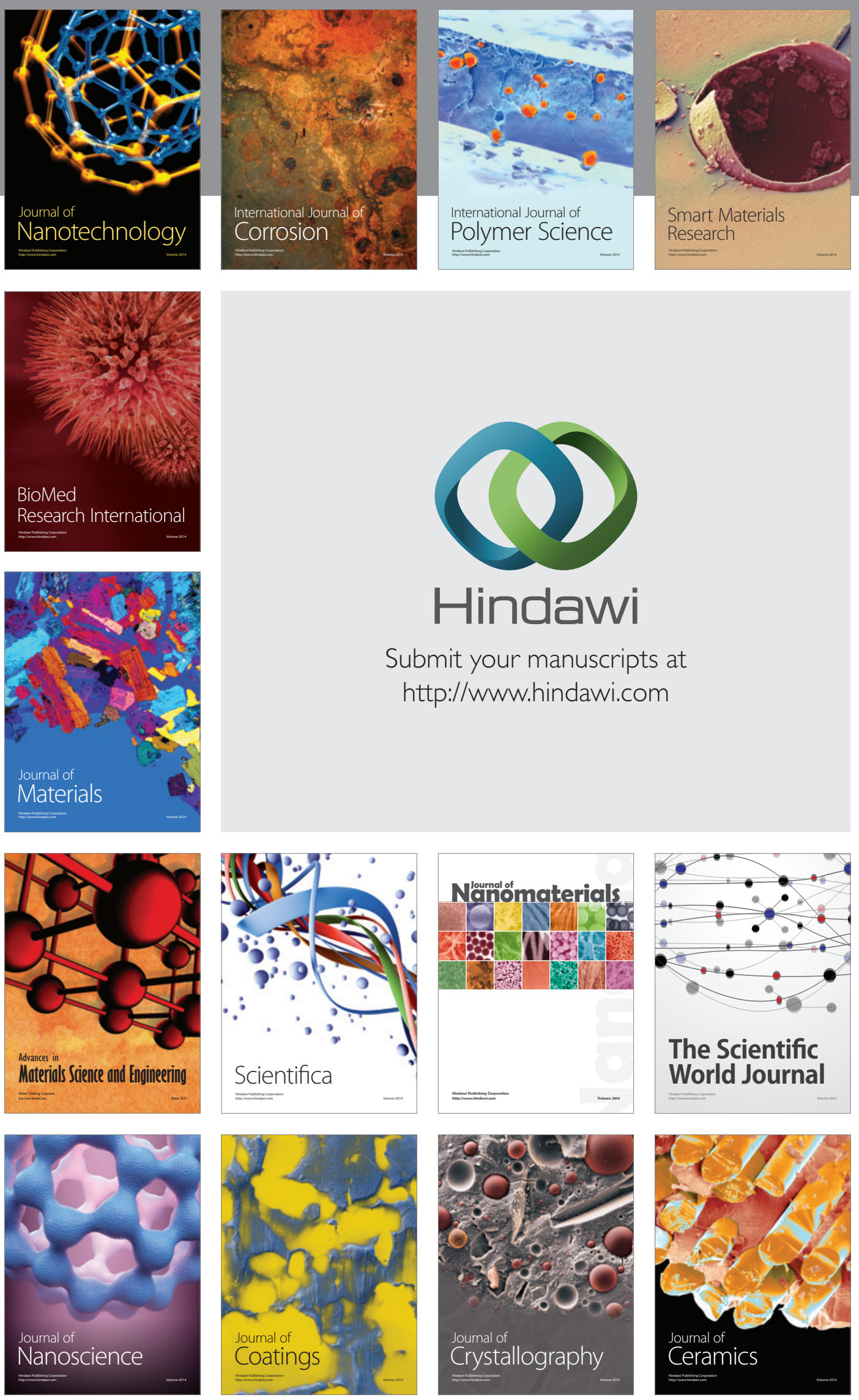

The Scientific World Journal

Submit your manuscripts at

http://www.hindawi.com

\section{World Journal}

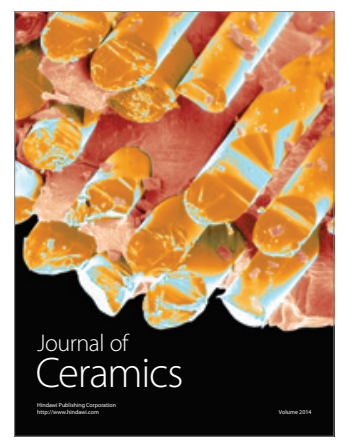

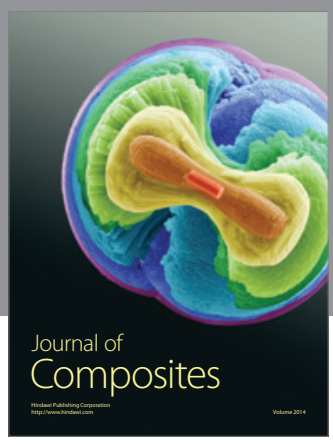
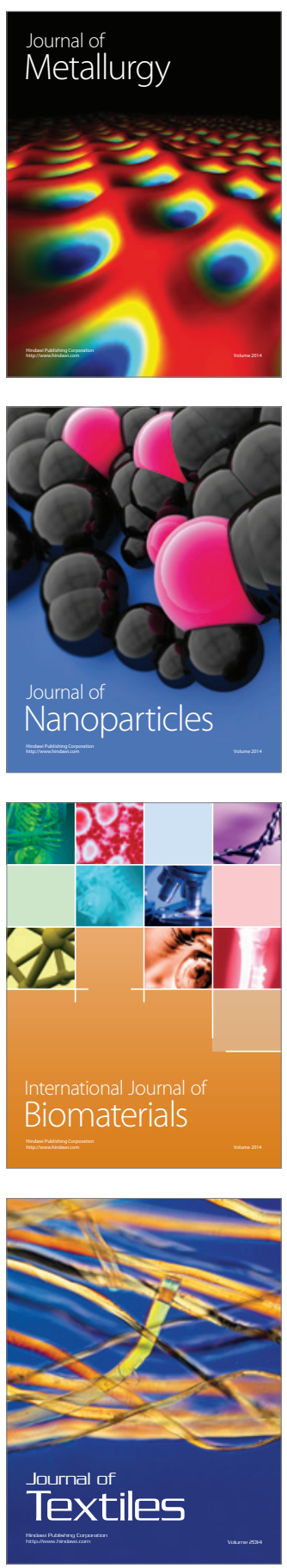